\title{
LAS RELACIONES DE GÉNERO EN EL CONTEXTO ESCOLAR. \\ UN ESTUDIO DE CASO A NIVEL DE EDUCACIÓN PREESCOLAR, COSTA RICA
}

\author{
Dra. Ana Lupita Chaves Salas \\ Directora e Investigadora IIMEC \\ Instituto de Investigación para el \\ Mejoramiento de la Educación Costarricense \\ Profesora Escuela de Formación Docente \\ Universidad de Costa Rica
}

\begin{abstract}
RESUMEN
Este artículo presenta una investigación cualitativa que se realizó en un salón de clase del nivel de educación preescolar, con el propósito de conocer cómo se producen las relaciones de género en el contexto escolar. En el estudio se encuentra que los estereotipos de género están presentes en la cotidianidad del aula y de diversas maneras.
\end{abstract}

Descriptores: Relaciones de género. Niñas y niños. Educación preescolar. Contexto escolar. Costa Rica. Siglo XX.

\section{INTRODUCCIÓN}

El niño y la niña al entrar en contacto con la cultura a la que pertenecen se apropian de los símbolos que son de origen social para posteriormente internalizarlos. En este proceso, los infantes se apropian de la cultura y construyen su identidad de género en las relaciones sociales, en la comunicación e interacción con los otros y las otras. 
Los primeros años de vida del ser humano son esenciales en su formación integral, puesto que el desarrollo de la inteligencia, la personalidad y el comportamiento social en las personas ocurre más rápido durante esos años (Rivero 1998). Toda experiencia vivida va moldeando la manera de ser, de pensar y de actuar de cada individuo, por ello los centros infantiles de educación inicial que atienden a niños y niñas desde los primeros meses hasta los seis años de vida, se convierten en instituciones fundamentales para la sociedad puesto que pueden contribuir a moldear identidades críticas, activas, autónomas, solidarias y creativas, o por el contrario identidades pasivas e individualistas.

Desde esta perspectiva, consideré importante realizar un estudio cualitativo en un salón de clase de educación inicial que me permitiera captar los significados que se transmiten en la actividad cotidiana del aula. En la investigación se analizan diferentes aspectos de la vida escolar, sin embargo en este artículo me ocupo de las relaciones de género que se propician entre estudiantes y educadora con el propósito de captar las subjetividades que ayudamos a construir en las interacciones diarias; y de esta forma tomar conciencia y reflexionar sobre el papel que desempeñamos en el salón de clase ya sea como educadoras o educadores que reproducimos conductas sexistas o que propiciamos relaciones equitativas y solidarias entre géneros.

\section{GÉNERO Y PROCESOS EDUCATIVOS}

El género es una construcción socio-cultural que asigna determinados comportamientos a hombres y a mujeres, y que los diferencia en términos de papeles y actividades que desarrollan en la sociedad, esta diferenciación que establece lo femenino y lo masculino, a la 
vez, va fortaleciendo jerarquías, entre unos y otras, es decir va estableciendo relaciones de poder y situaciones de inequidad entre ellos y ellas (Díaz 1999).

La identidad genérica se construye desde la concepción, el ser humano al relacionarse con su cultura aprende conductas que son de origen social para luego internalizarlas. En la apropiación de la cultura, las personas construyen su identidad en las relaciones sociales, en la comunicación e interacción con los otros y las otras. En consecuencia, es esencial develar y analizar las relaciones de poder que se generan en los contextos escolares para tomar conciencia de las identidades y subjetividades que se construyen en las interacciones del salón de clase.

La población estudiantil adquiere en la escuela diversos conocimientos y conductas como consecuencia del curriculum oficial y de los intercambios que se producen en el aula y en la institución. Muchas veces los contenidos del curriculum son poco pertinentes e impuestos por el sistema educativo, no interesan a la población estudiantil, por lo que son fácilmente olvidados, mientras que las relaciones sociales van, poco a poco, configurando subjetividades y modos de percibir el mundo. Al respecto José Gimeno y Ángel Pérez afirman:

“...el aprendizaje de los mecanismos, estrategias, normas y valores de interacción social que requiere el discurrir con éxito en la vida académica compleja y personal del grupo del aula y del centro van configurando paulatinamente representaciones y pautas de conducta que, extienden su valor y utilidad más allá del marco de la escuela. Esta va induciendo una forma de ser, pensar y actuar, tanto más válida y sutil cuanto más intenso sea el isomorfismo o similitud entre la vida social del aula y las relaciones sociales en el mundo del trabajo o en la vida pública..." (Gimeno y Pérez 1993: 22).

La vida del aula y del centro educativo son escenarios vivos de relaciones donde se intercambian directa o indirectamente ideas, valores e intereses diferentes mediante los 
procesos de comunicación. En este proceso es fundamental el papel que asume el lenguaje ya que mediante él, "...la sociedad inyecta en el individuo las significaciones que ha elaborado en el transcurso de su historia..." (Morales 1990: 11).

En la comunicación diaria, las personas van estableciendo una relación con las demás y van construyendo su identidad individual y social. En nuestra sociedad, el lenguaje regulado por los grupos hegemónicos transmite significados acordes con la ideología dominante; de acuerdo con Basil Bernstein:

“...Las relaciones de clase generan, distribuyen, reproducen y legitiman formas características de comunicación, que transmiten códigos dominantes y dominados, y esos códigos posicionan de forma diferenciada a los sujetos en el proceso de adquisición de los mismos..." (Bernstein 1994: 25)

De tal modo, en la relación social se lleva a cabo el control simbólico mediante el cual la conciencia adopta una forma especializada, a través de diferentes formas de comunicación, que transmite una determinada distribución de poder y las categorías culturales dominantes.

El lenguaje y la intercambio social ponen de manifiesto las desigualdades sociales en cuanto a género, clase social y grupo étnico, por tal razón es fundamental el uso consciente de la expresión oral y de los significados que se transmiten en el salón de clase.

Como consecuencia de que el poder ha estado históricamente en manos de los hombres y ellos han tenido la autoridad de conformar la cultura, el lenguaje como parte de la cultura determina que el género masculino designa al varón y a toda la especie humana, invisibilizando a las mujeres. Al respecto Alda Facio afirma: 
“...Si sólo los hombres han tenido el poder de definir, sólo ellos han conformado la cultura y por ende, esa cultura es masculina. En otras palabras, las mujeres como seres humanos plenos, no existimos en esta cultura..." (Facio 1994: 21).

Por lo tanto, el lenguaje no es neutro, ni en su uso ni en su estructura, refleja la situación social y contribuye a formar una visión de mundo. Los usos sexistas del lenguaje nos moldean nuestras percepciones y pensamientos sobre hombres y mujeres. El lenguaje crea subjetividades y fomenta la desigualdad de género, étnia y clase. De tal manera la identidad de un hombre o una mujer es producto de los procesos de socialización que se generan en el contexto socio cultural donde se desarrolla. Por ello es fundamental asumir una posición crítica y tomar conciencia sobre el uso del lenguaje que invisibiliza a la mujer y refleja una determinada forma de poder. Al respecto Rosa Santórum y Ramona Barrio afirman; que en el contexto socio-cultural actual

“...se aprende a ser mujeres y hombres también a través del lenguaje, hablando y escuchando a hablar y a través de mensajes encubiertos. Al utilizar la lengua como les enseñaron, niñas y niños mantienen y perpetúan el sexismo, la subordinación femenina y la transmisión de valores andrométricos..." (Santórum y Barrio 1998: 58).

Por lo tanto se debe tomar conciencia de esta realidad, a partir de la cual es posible actuar sobre ella y tender a modificarla mediante una acción pedagógica socialmente crítica, puesto que en los centros educativos esta diferenciación sexual se manifiesta en la cotidianidad, tanto en el lenguaje como en la interacción social, asimismo en el material didáctico y en los libros de texto lo que contribuye a mantener las diferencias de género, las relaciones de poder y la ideología dominante.

Con el propósito de analizar las relaciones de género que se producen en el contexto escolar decidí realizar una investigación cualitativa que me permitió comprender e interpretar 
las acciones humanas en su contexto real. En el siguiente apartado resumo la metodología que seguí para realizar el estudio.

\section{EL CAMINO RECORRIDO}

La investigación la realicé interactuando con un grupo de niñas y niños en edades comprendidas entre los cinco años y tres meses, y los siete años, y una educadora en un centro educativo público de zona urbana de San José. Las observaciones las efectué durante el curso lectivo de 1999 y las registré mediante el diario de campo, las grabaciones y los videos. También utilicé la entrevista, el relato de experiencia y el análisis de documentos con propósito de captar los significados que no emergen fácilmente, sino que están ocultos pero que son fundamentales para interpretar y conocer la realidad-realidades del aula.

La pregunta que orientó el proceso investigativo, en relación con este tema, fue la siguiente:

1. ¿Cómo se dan las relaciones de género en el salón de clase ?

La estrategia de investigación fue inductiva y progresiva, me incliné por el método hermenéutico-dialéctico para comprender e interpretar los significados que se transmitieron en las interacciones del aula (Martínez 1989).

El proceso investigativo lo fui desarrollando en las siguientes etapas:

I. Reflexiones individuales

II. Elaboración teórica

III. Reflexiones colectivas 
IV. Identificación del tema

V. Negociación de entrada

VI. Inmersión al salón de clase

VII. Compartiendo la información

VIII. Análisis de datos

Estas etapas no se dieron de manera lineal sino que la mayoría de ellas estuvieron presentes durante todo el proceso investigativo.

Las observaciones las hice de la siguiente manera:

- Una o dos veces por semana en los meses de febrero, marzo y abril, en el mes de mayo hice un primer acercamiento al análisis de la información obtenida hasta ese momento.

- Una o dos veces por semana durante los meses de junio, julio y agosto

- En agosto ingresó al salón de clase una estudiante que realizó su práctica profesional durante el segundo semestre, por tal situación, observé las interacciones del aula únicamente cuando la educadora era la que dirigía el trabajo con los niños y las niñas, es decir una o dos veces por semana, cada quince días.

- En setiembre y octubre no visité la institución.

- Una vez por quincena durante los meses de noviembre y diciembre.

Las observaciones las efectué en períodos de dos horas aproximadamente y seleccioné los momentos de la jornada diaria más relevantes para el propósito del estudio: actividades iniciales, conversación, juego en áreas, educación física y merienda. 
El análisis de la información lo realicé de manera progresiva, en primer término leía los datos y reflexionaba sobre lo vivido para tratar de comprender lo que ocurría en el contexto del aula, transcribía las observaciones, y agregaba mi percepción sobre ellas, posteriormente comentaba las notas con la educadora e incluía sus sugerencias con el fin de encontrar el significado de la práctica educativa y por último agregaba notas teóricas.

El análisis fue un proceso profundo, dinámico y sistemático que requirió muchas horas de reflexión y de diálogo para descubrir ese significado subjetivo que orientaba las actuaciones de las personas en el contexto natural del aula.

Para profundizar en el significado de la acción humana en el salón de clase analicé otras fuentes: el planeamiento, los trabajos de los niños y las niñas, el material didáctico que utilizaba la educadora, las evaluaciones, un relato de experiencia que elaboró la educadora, las notas de las conversaciones informarles y las entrevistas realizadas en el transcurso del año.

Toda esa información, fue consultada una y otra vez en el proceso de análisis. Con base en toda esos datos definí la siguiente área y sub-áreas sobre las relaciones de género que se desarrollaron en el contexto escolar:

\section{Poder, lenguaje y género}

- Lenguaje y participación

- Gustos e intereses

- Material literario

Con el área y sub-áreas definidas elaboré matrices con cuatro columnas para organizar la información donde incluía las notas del diario, documentos, entrevistas y comentarios de la educadora, mis percepciones y las notas teóricas. Estas matrices se constituyeron en un excelente instrumento para validar la información obtenida, ordenar los datos y realizar el 
análisis, sin embargo siempre recurrí a las fuentes originales: mi diario, las entrevistas, los diálogos, los videos, el relato de experiencia, la bibliografía, las carpetas de los niñas y los niños, etc., por lo que estas matrices no fueron estáticas sino muy dinámicas, ya que muchas veces encontré elementos nuevos para enriquecerlas.

Seguidamente presento un ejemplo de la matriz:

\begin{tabular}{|c|c|c|c|}
\hline \multicolumn{4}{|c|}{ Área: Poder, lenguaje y género Subárea: Material literario } \\
\hline Notas de campo & $\begin{array}{l}\text { Documentos, } \\
\text { entrevista } \\
\text { educadora }\end{array}$ & Percepciones personales & $\begin{array}{l}\text { Notas } \\
\text { teóricas }\end{array}$ \\
\hline $\begin{array}{l}\text { E: - Niños estamos listos para escuchar el } \\
\text { cuento. El cuento tiene una enseñanza muy } \\
\text { bonita, ustedes van a poner atención y me la } \\
\text { van a contar. } \\
\text { La educadora narra el cuento "El conejo y la } \\
\text { tortuga". Es un libro con láminas grandes. } \\
\text { E: -¿Les gustó el cuento? } \\
\text { Niños y niñas:- Sí. } \\
\text { Educadora- ¿Qué pasó en el cuento? } \\
\text { Niño: -Que la tortuga ganó. } \\
\text { Ana María - El conejo aprendió a no burlarse. } \\
\text { E: - y ¿Por qué no hay que burlarse? } \\
\text { Paola: - Porque es muy feo. } \\
\text { E:-¿Por qué es muy feo? } \\
\text { Paola: - Porque uno se puede sentir muy mal. } \\
\text { E:- ¿Por qué ganó la tortuga? } \\
\text { Erick:- Porque el conejo se quedó dormido. } \\
\text { E: ¿Por qué más? } \\
\text { Johana: -Porque la tortuga se esforzó más. } \\
\text { E: -Sí, porque ella era muy lenta porque tenía } \\
\text { que cargar su casita. } \\
\text { Nicole - Uno no se puede burlar de la gente } \\
\text { porque la acusa con la mamá. } \\
\text { E: -Sólo por eso. } \\
\text { Diana: -No, porque nos sentimos mal. } \\
\text { Pablo: - No hay que burlarse de los amigos. } \\
\text { E: -Si ustedes fueran la tortuga que ganó } \\
\text { ¿Cómo se sentirían? } \\
\text { Niños y niñas -Bien } \\
\text { Educadora:- Si fueran la tortuga ¿Qué le } \\
\text { hubieran dicho al conejo? } \\
\text { Nicole: - Si no se hubiera dormido, hubiera } \\
\text { ganado. } \\
\text { Dyana: - ¿Por qué se burlaba? Que como él }\end{array}$ & 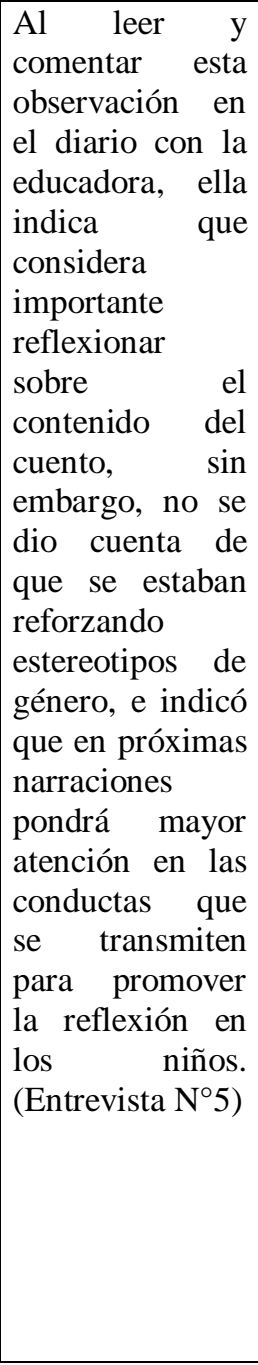 & $\begin{array}{l}\text { En esta situación, la } \\
\text { educadora cuestiona a los } \\
\text { infantes en relación con el } \\
\text { contenido del cuento y los } \\
\text { induce a reflexionar y a } \\
\text { criticar sobre lo que } \\
\text { sucedió. En el diálogo, } \\
\text { emergen todo tipo de } \\
\text { explicaciones desde que no } \\
\text { hay que burlarse "porque lo } \\
\text { acusa con la mamá" donde } \\
\text { sobresale la preocupación } \\
\text { por la autoridad, hasta la } \\
\text { que indica "que uno puede } \\
\text { sentirse mal" donde lo } \\
\text { importante es la persona y } \\
\text { sus sentimientos. En esta } \\
\text { interacción, me pareció } \\
\text { importante que la educadora } \\
\text { incentivara lá } \\
\text { reconstrucción crítica de lo } \\
\text { que sucedió en el cuento, } \\
\text { sin embargo, ella no estaba } \\
\text { conciente de los } \\
\text { significados que transmitía } \\
\text { la narración en cuanto a } \\
\text { estereotipos de género. } \\
\text { Desde esta perspectiva, la } \\
\text { narración refuerza los } \\
\text { estereotipos presentes en } \\
\text { nuestro contexto social, el } \\
\text { conejo es veloz, activo, } \\
\text { decidido, reta a la tortuga a } \\
\text { correr, ella es lenta, pasiva, }\end{array}$ & $\begin{array}{l}\text { Al respecto } \\
\text { Díaz 1999:10 } \\
\text { indica que el } \\
\text { material } \\
\text { educativo } \\
\text { transmite } \\
\text { estereotipos } \\
\text { de género, } \\
\text { que muchas } \\
\text { veces, no son } \\
\text { percibidos } \\
\text { por el } \\
\text { personal } \\
\text { docente, } \\
\text { porque han } \\
\text { internalizado } \\
\text { la cultura } \\
\text { hegemónica y } \\
\text { ven como } \\
\text { natural el } \\
\text { orden en } \\
\text { establecido y } \\
\text { las } \\
\text { desigualdades } \\
\text { sociales y } \\
\text { económicas. }\end{array}$ \\
\hline
\end{tabular}




\section{- Lenguaje y participación}

En el lenguaje oral cotidiano se evidencian una gran cantidad de códigos que omiten lo femenino. En el vocabulario de los niños, las niñas y la educadora se invisibilizaba a la mujer puesto que, en la mayoría de las veces, se utilizaba el género masculino en la comunicación diaria. Este lenguaje se usó en un salón de clase donde habían 18 niñas y 14 niños. Por ejemplo:

E:- Niños a lavarse las manos.

E:- ¿Quién cree que puede leer la fecha solito, solito?

E: - Niños vamos a educación física.

Según Bernstein (1998), el lenguaje sexista es producto de la influencia de la ideología dominante que introyecta en todos y todas, costumbres, creencias y valores. De esta forma, se va legitimando el orden establecido mediante el lenguaje de manera inconsciente, lo que contribuye a transmitir y reproducir la cultura dominante y las diferencias por género, con una función dirigida al control simbólico y social.

Durante las primeras observaciones que realicé en el aula, se evidenció que los varones participaban con mayor frecuencia durante los diferentes momentos de la jornada escolar, se les daba en mayor medida la palabra y eran los que demostraban algún ejercicio para que sus compañeras y sus compañeros lo repitieran. Sin embargo, cuando la educadora solicitaba apoyo para limpiar las mesas, recoger la basura, o barrer el aula; las niñas se ofrecían en 
mayor medida que los niños, pero en estas situaciones la profesora seleccionaba estudiantes de ambos sexos para desempeñar dicha labor.

Estas situaciones las analizábamos en las entrevistas con la educadora, reflexionábamos del por qué se presentaban, y poco a poco, la participación de los niños y las niñas en el salón de clase fue más equitativa.

En cuanto al lenguaje sexista, también se produjeron cambios y la educadora empezó a utilizar el género femenino en su lenguaje oral y escrito, y en los materiales que elaboraba para las estudiantes y los estudiantes:

E: -Niños y niñas a recoger.

E:- Los niños y las niñas que jugaron en esta mesa deben limpiarla.

El cambio se produjo a partir del mes de agosto, pero no fue un cambio radical, pero, al menos, se inició un proceso de transformación en el uso del lenguaje oral y escrito.

\section{- Gustos e intereses}

Fue interesante captar como las conductas sexistas de nuestra cultura patriarcal se reflejan hasta en lo que los niños y las niñas saben sobre algún tema, así por ejemplo, la educadora en una ocasión preguntó ¿qué saben sobre las mariposas? Las niñas sabían que las mariposas eran de colores, ponían huevos y tenían hijos, y los niños que las mariposas volaban, y que "son gusanos que están en capullo que se rompe y sale la mariposa". En este ejemplo se evidencia claramente cómo la cultura machista es captada e internalizada por las 
personas desde los primeros años de vida, de tal manera el contenido o los intereses sobre algún tema son diferentes según su sexo. Estas diferencias genéricas también las percibí en los dibujos, las niñas hacían con mayor frecuencia flores, casas, muñecos; y los niños carros, aviones, barcos, etc.

Durante el tiempo que observé el aula, fue muy evidente como los niños y las niñas representaban acciones, actitudes, creencias e ideas estereotipadas en cuanto al comportamiento de hombres y de mujeres en la sociedad, comportamientos que han internalizando en el proceso de socialización.

En las áreas de juego, las niñas escogían en mayor medida el área de lenguaje, dramatización y artes; los niños construcción (bloques, legos, mecanos), ciencias, matemática y arena. En el área de dramatización, las niñas eran mamás, cocinaban, limpiaban, iban de compras; algunas representaban doble función eran amas de casa y trabajan también fuera del hogar, los niños jugaban de doctores y arreglaban el techo, la cocina, la refrigeradora.

\section{Fotos aquí.}

Como lo indica Díaz (1999) en éstos comportamientos sexistas se va subvalorando un sexo sobre otro y se va promoviendo la desigualdad jerárquica entre ellos y ellas, de tal forma las mujeres asumen un papel de subordinación y los hombres un papel dominante, como consecuencia de nuestra cultura patriarcal, pero en este intercambio, al mismo tiempo, se va discriminando a los varones ya que se les excluye de actividades del hogar y se les va identificando con actitudes de autoritarismo y de insensibilidad. Estas acciones traen 
"...consecuencias negativas para todos los individuos, hombres y mujeres, porque limitan sus posibilidades como personas y les niega determinados comportamientos..." (Subirats 1994: 61 citado por Díaz 1999: 9).

\section{- Material literario}

En las observaciones que realicé fue notorio que la literatura utilizada (cuentos, poesías, rimas y canciones) presentaban marcados estereotipos de género.

Los cuentos, las canciones y las poesías que escuché en el salón de clase, en su mayoría, llevaban una gran carga ideológica a favor de la reproducción de conductas sexistas. En algunos de ellos, no existía o era muy escasa la presencia femenina como el cuento "Yo soy yo", en la poesía "El payaso del viento", "El flautista de Hamelín”, "Pinocho", "El gato con botas", "Los dos ratones"; en las canciones "Pedro comió pan", "Los esqueletos", "Que todos los niños estén muy atentos", "El payaso se pinchó la nariz", "Los diez pececitos".

En otras ocasiones a las mujeres se les mencionaba en situaciones de inferioridad social o subordinación: "Caperucita Roja" , "La Bella Durmiente", "El mono Tulín", "El conejo y la tortuga", entre otros. La literatura presentaba frases desvalorizantes con las cuales se evidencia la superioridad del sexo masculino y la inferioridad del sexo femenino. También se utilizan términos genéricos y plurales masculinos que invisibilizan la presencia femenina.

En las ilustraciones se presentan con mayor frecuencia figuras masculina en relación con los personajes femeninos, y ellos aparecen en primer plano. Esto evidencia, una vez más, la carga ideológica que transmiten los diferentes recursos educativos que usamos en la escuela y 
que van legitimando diferentes niveles de poder y papeles específicos según el sexo de las personas.

En el salón de clase, la educadora invitaba a los niños y a las niñas a conversar sobre el contenido de los cuentos, canciones y poesías, sin embargo, no hacían una reflexión crítica de las conductas sexistas, creo que ella, al iniciar el curso lectivo, no estaba conciente de los significados que transmitían las narraciones en cuanto a estereotipos de género. Posteriormente, al analizar las notas de campo, los vídeos y la literatura, se dio un cambio en la educadora y empezó a seleccionar con mayor cuidado el material literario, y hacer preguntas y comentarios sobre posibilidades de realización que tenemos los seres humanos sin

distinción de género. En este proceso fue lamentable darnos cuenta que el mercado está inundado de literatura sexista, y que es realmente difícil encontrar material que promueva la equidad de género.

\section{REFLEXIONES FINALES}

Ante estos hechos ¿Qué podemos hacer las educadoras y los educadores para transformar esta realidad?

Creo que es fundamental tomar conciencia de que todos y todas somos reproductores de desigualdades, con nuestro lenguaje sexista establecemos jerarquías entre las personas y nos convertimos en instrumentos de la cultura patriarcal que subestima e invisibiliza a las mujeres, por tal razón es fundamental promover procesos de autorreflexión en educadores y educadoras para que estemos concientes de lo que hacemos, de lo que decimos, de los significados que 
transmitimos en la interrelación con los demás. Para ello, es preciso descubrirnos, es decir, analizar nuestra identidad, nuestras creencias y nuestros pensamientos.

Lo importante es conocernos, descubrir y develar los símbolos que transmitimos para transformarlos. En la medida que seamos concientes de la influencia que tiene la cultura hegemónica en nuestra manera de ser y de ver el mundo, en esa misma medida podremos contrarrestar la influencia de la ideología dominante.

Es urgente crear nuevas formas de relacionarnos con los otros y las otras y construir juntos una práctica educativa democrática, humanista, no sexista y con equidad genérica.

Para lograr una transformación en la acción educativa y una nueva cultura de género, creo importante, también, una transformación en las instituciones formadoras de docentes, dirigida a:

- Promover en los estudiantes y las estudiantes procesos de autorreflexión para que se cuestionen sobre su papel como educadores y educadoras dentro de la sociedad, sobre el tipo de ser humano que desean formar y sobre la sociedad que quieren ayudar a construir .

- Es fundamental promover que las estudiantes y los estudiantes realicen prácticas en el aula donde analicen los procesos de socialización y las relaciones de poder que se dan en el intercambio escolar, y de esta manera vivencien las prácticas sexistas y descubran la influencia que tiene la educadora o el educador en la construcción de la identidad y subjetividad de las estudiantes y los estudiantes. Creo que para que se dé el cambio, primero se debe vivenciar la situación, analizarla críticamente y de esta forma tomar conciencia para iniciar procesos de transformación.

- Es preciso que la formación de educadores y educadoras tenga un enfoque más sociopolítico, para que no seamos utilizados como instrumentos de la ideología dominante y 
no seamos reproductores, inconsciente e ingenuos, de desigualdades de todo tipo. En la actualidad la formación se dirige más a un enfoque psicologista de la educación, énfasis que debemos cambiar.

- Analizar críticamente el material educativo que usamos en nuestras aulas y en todos los niveles, pues están cargados de mensajes sexistas que profundizan las desigualdades entre hombres y mujeres.

En síntesis, lo importante es

“...construir una nueva cultura del género que se base en la solidaridad entre hombres y mujeres como principio ético-político, que implique el reconocimiento del otro, de la otra, la defensa de la libertad y del poder personal y grupal para ambos géneros, la eliminación de jerarquías de un género sobre otro y la comprensión de todos y todas como ciudadanos del mundo, sin distinción de género, clase, región, nacionalidad o raza..." (Lagarde,1999).

\section{BIBLIOGRAFÍA}

Acker, Sandra. 1995. Género y educación. Madrid: NARCEA S.A.

Bernstein, Basil. 1994. La estructura del discurso pedagógico Madrid: Morata. . 1998. Pedagogía, control simbólico e identidad. Madrid: Morata.

Díaz, María Isabel. 1999. "Algunas reflexiones acerca de: la dimensión de género en el curriculum de la educación parvularia". En TEMAS PEDAGÓGICOS. Serie de Cuadernillos de Estudio. Santiago de Chile: Junta Nacional de Jardines.

Facio, Alda. 1994. "Cuando el género suena, cambios trae". Introducción al análisis de género: categorías analíticas básicas. Managua: Programa Interdisciplinario de Estudios de Género, Universidad Centroamericana.

Gimeno, José y Pérez, Ángel. 1993. Comprender y Transformar La Enseñanza. $2^{\mathrm{a}}$ ed. Madrid: Morata S.A.

Lagarde, Marcela. 1999. Una mirada en el umbral del milenio. Heredia: Instituto de Estudios de la Mujer, Universidad Nacional.

Morales, Pedro. 1990. El papel del lenguaje en el desarrollo cognoscitivo: anteposición de la perspectiva piagetiana frente a la perspectiva soviética. San Juan-Puerto Rico: Recinto de Río Piedras. Universidad de Puerto Rico, mimeografiado. 
Rivero, José. 1998. "La educación infantil en el siglo XXI”. Proyecto Principal de Educación en América Latina y el Caribe. Boletín 47. Santiago de Chile: UNESCO, Oficina Regional de Educación para América Latina y el Caribe.

Santórum, Rosa y Ramona Barrio. 1998. "Del sexismo lingüístico a la diferenciación sexual en el lenguaje". Desarrollo del lenguaje y cognición. Madrid: Pirámide. 